\title{
Vojta Therapy for a 12 year-old Child with Cerebral Palsy
}

\author{
Ewa Gajewska ${ }^{1)}$, Barbara Neukirch ${ }^{2)}$ \\ 1) Department of Physiotherapy, Rheumatology and Rehabilitation, Karol Marcinkowski University of \\ Medical Sciences: Ul. 28. Czerwca 1956r. 135/147, 61-545 Poznan, Poland. \\ TEL:+4861-8310-244,FAX: +4861-8310-244,E-mail: karasinska1@wp.pl \\ 2) Clinic of Rehabilitation, Aachen, Germany
}

\begin{abstract}
Purpose] The aim of the study was to investigate the effect of Vojta therapy on motor improvement in an older child with cerebral palsy. [Subjects] Vojta therapy was performed for a 12 year-old child with cerebral palsy who had significantly arrested motor development during the first years of life. [Methods] The treatment was applied in two separate therapeutic units of 14 and 6 weeks, respectively, which were separated by an 8-month Vojta-free period. Therapeutic progress was gauged by the gross motor function measure, the gross motor function classification system, and the manual ability classification system. [Results] The gross motor function measure improved significantly following the first therapeutic unit, then decreased during the interval, but improved again following the second therapeutic unit. In the gross motor function classification system, the patient was classified as level III and this remained unchanged, despite great progress in specific motor functions. In the manual ability classification system assessment the child was classified as level III during the entire assessment period. [Conclusion] Using Vojta therapy can achieve dynamic locomotor and gross motor development in older cerebral palsy patients. Key words: Vojta therapy, Reflex locomotion, Cerebral palsy
\end{abstract}

(This article was submitted Jan. 16, 2012, and was accepted Mar. 13, 2012)

\section{INTRODUCTION}

Cerebral palsy (CP) is a medical term, which encompasses a wide spectrum of syndromes relating to the early onset of posture and motor impairment ${ }^{1)}$. Cerebral palsy has been reported to be the most common cause of physical disability in children ${ }^{2}$. At present, various rehabilitative approaches are available for improving the postural control, balance and locomotor function of children with cerebral palsy. Vojta therapy is one such approach. It relies on the principle of isometric strengthening via tactile stimulation ${ }^{3)}$. This encourages the development of normal movement patterns through the activation of inherent global motor complexes. Whilst it is generally considered that Vojta therapy can be successfully used to treat young infants, less is known about its suitability for older children with cerebral palsy ${ }^{3,4)}$. It was with this in mind that we report a case in which Vojta therapy was successfully used to treat a 12 year-old child with cerebral palsy.

\section{CASE REPORT}

The motor development of a 12 year-old female patient during the first years of her life was significantly arrested. At the age of four, the patient was able to perform homologous crawling. After three years, numerous upper and lower extremity contractures developed and the motor status of the patient regressed. At 8 years of age, the patient underwent
4 months of intensive physiotherapy and also attended hippotherapy. Passive verticalisation was also performed. However, neither functional improvement nor progression in independence was achieved. The patient was mentally healthy. At the age of 12 years, the patient, diagnosed as having spastic quadriplegic cerebral palsy $(\mathrm{CP})$ in her home country, was first assessed by a Vojta therapist in Germany. Due to cultural differences, ADL was not assessed.

\section{METHODS}

The patient underwent Vojta therapy twice a day (45'), five days a week. The rehabilitation consisted of two separate therapeutic programs of 14 and 6 weeks, respectively, which were separated by an 8-month Vojta-free interval. During this interval, standard kinesis therapy was performed. The patient's locomotor development was evaluated at the beginning and at the end of each respective Vojta therapy unit.

The following methodologies were utilized to gauge therapeutic progress: the gross motor function measure (GMFM-88) the gross motor function classification system (GMFCS) and the manual ability classification system (MACS). In addition, for the GMFM-88 assessment, specially design software ${ }^{5)}$ was used to measure and analyze 66 selective individual locomotive functionalities. This was performed in accordance with the classic and readily accepted form of GMFM-88, namely GMFM-66. According 
Table 1. Grouped gross motor elements and the relative assessment scores according to the GMFM-88 evaluation method

\begin{tabular}{lcccc}
\hline Gross motor element & 1st assessment & 2nd assessment & 3rd assessment & 4th assessment \\
\hline lying \& rolling & 33.33 & 78.43 & 82.35 & 92.16 \\
sitting & 6.67 & 40.00 & 35.00 & 40.00 \\
crawling \& kneeling & 2.38 & 19.05 & 7.14 & 26.19 \\
standing & 0.00 & 35.90 & 10.26 & 48.72 \\
walking, running \& jumping & 0.00 & 9.72 & 0.00 & 16.67 \\
total score & 8.48 & 36.62 & 26.95 & 44.75 \\
\hline
\end{tabular}

Assessment scores are measured in percentage

to GMFCS, the patient was initially classified as level V and an additional assessment indicated level IV on the MACS assessment scale.

The main locomotive-related pathologies of the patient were identified and therapeutic aims were selected. A long-term objective of the treatment protocol was attainment of bipedal locomotion by the patient.

The Vojta technique requires the patient to be orientated in a number of different predefined initial positions in order to evoke appropriate innate motor complexes. As this was not always feasible due to the presence of a large number of significant joint contractures, a number of modifications of the initial orientations were utilized ${ }^{6,7)}$. However, after two weeks of Vojta therapy, it was possible to orientate the patient in all initial positions included in reflexive rolling and reflexive crawling ${ }^{6}$. By activating different reflex zones, the functional differentiation of various autochthonic muscle groups took place.

\section{RESULTS}

The results of the GMFM- 88 assessment are shown in Table 1 . The GMFM- 88 total score improved significantly following the first therapeutic unit (the greatest progress), then decreased during the interval but improved again following the second therapeutic unit relative to the initial functionality score. During the 8-month interval, when Vojta therapy was not applied, the functional locomotive development appeared to decline.

In the GMFCS assessment the patient was classified as level III immediately following the first therapeutic unit and this remained unchanged, despite great progress in specific motor functions. In the MACS assessment the child was classified as level III during the entire assessment period. The results of the GMFM-66 assessment are shown in Figures 1 and 2.

Though ADL was not assessed, after the second therapy the patient was found able to go to school.

\section{DISCUSSION}

This clinical case report illustrates that proper rehabilitative measures can achieve dynamic locomotor and gross motor development in a relatively short period of time. It may be argued that since the motor functionality condition of the patient appeared to be neglected for a relatively long period of time, again due to cultural conditions, this may go some way in explaining the dramatic results, which were obtained. Nonetheless, to the best of our knowledge, this represents one of the very few studies to achieve such an improvement in an older child with cerebral palsy via the clinical use of Vojta therapy. The patient was able to successfully achieve bipedal mobility albeit with support. In addition, a number of other locomotive functionalities were

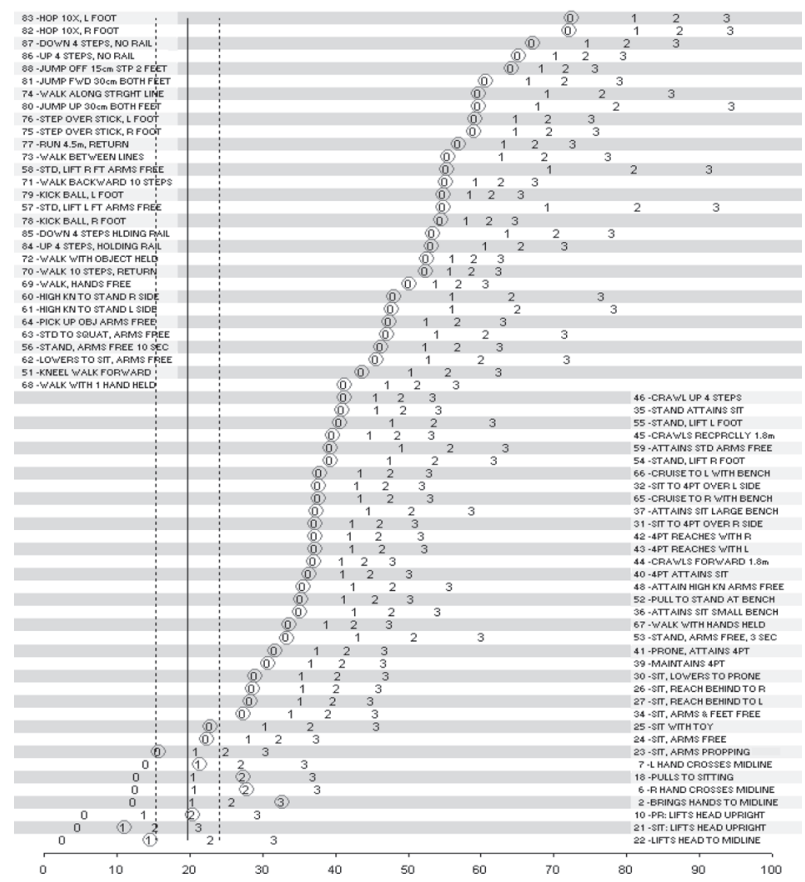

Fig. 1. Assessment of 66 selective individual locomotive functionalities for the GMFM-66 locomotive functionality classification prior to the initiation of Vojta therapy. *The left upper and right lower corner represent advanced and basic itemized activities, respectively. Red circlesdenote the assessed locomotive score. The vertical and dashed linesrepresent the mean $\%$ score with the associated confidence interval, respectively. 


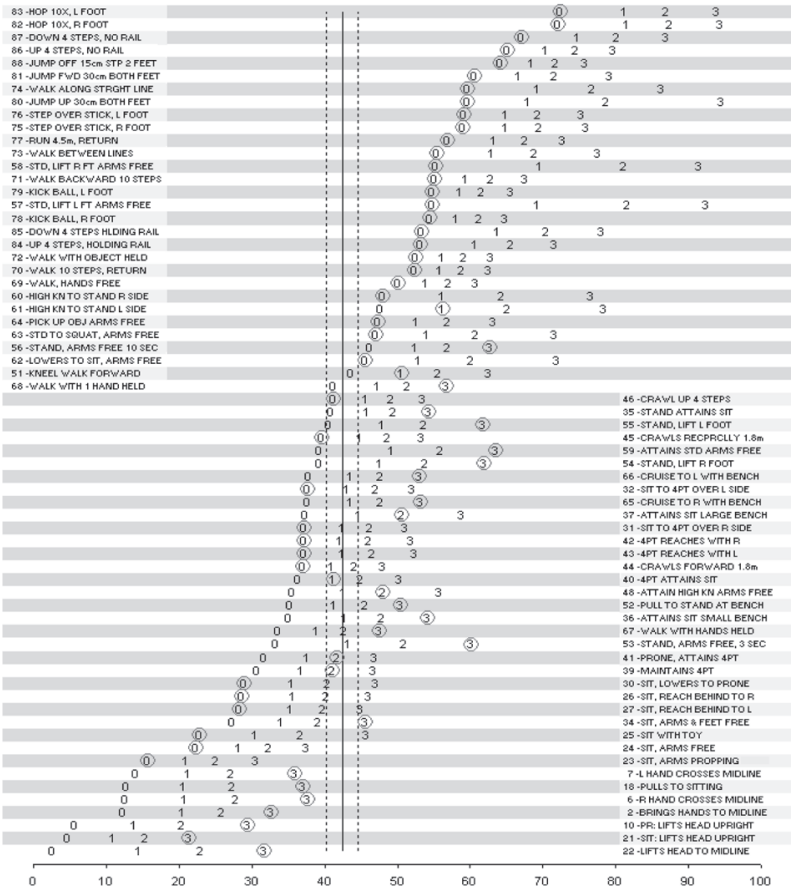

Fig. 2. Assessment of 66 selective individual locomotive functionalities for the GMFM-66 locomotive functionality classification immediately following the second therapeutic unit of Vojta therapy. The left upper and right lower corner represent advanced and basic itemized activities, respectively. Red circlesdenote the assessed locomotive score. The vertical and dashed linesrepresent the mean \% score with the associated confidence interval, respectively. The change in the mean \% locomotive score prior to and immediately following Vojta therapy is evident.

gained, thereby increasing the patient's independence. The total score of the GMFM-88 assessment increased markedly. In addition, in this relatively older child, who was initially apedal, improvements in antigravity mechanisms and motor coordination occurred. Whether or not this was due to her intellectual level, remains unclear. This stands in contrast to a study undertaken by Harries and Notorshvili $\left.{ }^{8}\right)$ of $106 \mathrm{CP}$ children, in which they found that the gross motor development reached a plateau at the age of 6-7 years.

In the current study, the observed spasticity was not inhibited by surgical means ${ }^{9)}$. Apart from a limited number of cases previously reported by Vojta ${ }^{10)}$, to the best of our knowledge this case report is probably one of the few to demonstrate improvement in locomotor function which may have been attained through the use of Vojta therapy during the relatively late rehabilitation of a child with cerebral palsy.

The application of Vojta therapy allowed for the development of higher motor function and intentional motor activities, thus increasing the independence of the patient.

\section{REFERENCES}

1) Koman LA, Smith BP, Shilt JS: Cerebral palsy. Lancet, 2004, 363: 1619-1631. [Medline] [CrossRef]

2) Reddihough DS, Collins KJ: The epidemiology and causes of cerebral palsy. Aust J Physiother, 2003, 49: 7-12. [Medline]

3) Giannantonio C, Papacci P, Ciarniello R, et al.: Chest physiotherapy in preterm infants with lung diseases. Ital J Pediatr, 2010, 36: 65. [Medline] [CrossRef]

4) Jóźwiak S, Podogrodzki J: Application and comparison of NDT-Bobath and Vojta methods in treatment of selected pathologies of the nervous system in children. Przegl Lek, 2010, 67: 64-66 (In Polish). [Medline]

5) Russel DJ: Avery LM, Rosenbaum PL, et al.: Gross Motor Function Measure (GMFM-66 and GMFM-88) User's Manual. London: Mackeith Press, 2002 .

6) Vojta V, Peters A: Das Vojta Prinzip. Verlag: Springer, 2007, pp 41-60.

7) Soo B, Howard JJ, Boyd RN, et al.: Hip displacement in cerebral palsy. J Bone Joint Surg Am, 2006, 88: 121-129. [Medline] [CrossRef]

8) Harries N, Kassirer M, Amichai T, et al.: Changes over years in gross motor function of 3-8 year old children with cerebral palsy: using the Gross Motor Function Measure (GMFM-88). Isr Med Assoc J, 2004, 6: 408-411. [Medline]

9) Manikowska F, Jóźwiak M, Idzior M: Muscle spasticity and functional status of children with cerebral palsy. Neurologia dziecięca, 2009,18: 31-36 (In Polish).

10) Vojta V: ZurPrognose der spätbehandeltencerebralparetischen Kinder. Kinderarzt, 1987, 18: 1161-1172. 